\title{
The influence of arm composition on the self-assembly of low-functionality telechelic star polymers in dilute solutions
}

\author{
Esmaeel Moghimi ${ }^{1,2} \cdot$ lurii Chubak $^{3} \cdot$ Dimitra Founta $^{1,2} \cdot$ Konstantinos Ntetsikas $^{4} \cdot$ George Polymeropoulos $^{4}$. \\ Nikos Hadjichristidis ${ }^{4}$. Christos N. Likos ${ }^{3}$. Dimitris Vlassopoulos ${ }^{1,2}$
}

Received: 13 July 2020 / Revised: 15 August 2020 / Accepted: 23 August 2020 / Published online: 25 September 2020

(C) The Author(s) 2020

\begin{abstract}
We combine synthesis, physical experiments, and computer simulations to investigate self-assembly patterns of lowfunctionality telechelic star polymers (TSPs) in dilute solutions. In particular, in this work, we focus on the effect of the arm composition and length on the static and dynamic properties of TSPs, whose terminal blocks are subject to worsening solvent quality upon reducing the temperature. We find two populations, single stars and clusters, that emerge upon worsening the solvent quality of the outer block. For both types of populations, their spatial extent decreases with temperature, with the specific details (such as temperature at which the minimal size is reached) depending on the coupling between inter- and intra-molecular associations as well as their strength. The experimental results are in very good qualitative agreement with coarse-grained simulations, which offer insights into the mechanism of thermoresponsive behavior of this class of materials.
\end{abstract}

Keywords Gels · Micelles · Nanoparticles · Polymer brushes · Polymer synthesis · Properties $\cdot$ Mechanical $\cdot$ Properties · Thermal $\cdot$ Self-assembly

\section{Introduction}

Self-organization of building blocks due to external stimuli is ubiquitous in most materials and all living organisms in nature. Inspired by this, a remarkable body of work has been performed to understand and emulate their response to temperature [1-3], $\mathrm{pH}$ [4-6] and light [7, 8], enabling

E. M. and I. C. contributed equally.

Christos N. Likos

christos.likos@univie.ac.at

Dimitris Vlassopoulos

dvlasso@iesl.forth.gr

1 Institute of Electronic Structure and Laser, FORTH, 71110, Heraklion, Crete, Greece

2 Department of Materials Science, Technology, University of Crete, 71003, Heraklion, Crete, Greece

3 Faculty of Physics, University of Vienna, Boltzmanngasse 5, A-1090, Vienna, Austria

4 Physical Sciences and Engineering Division, KAUST Catalysis Center, Polymer Synthesis Laboratory, King Abdullah University of Science and Technology (KAUST), Thuwal, 23955, Kingdom of Saudi Arabia the controlled design of their structure assembly. Recent advances in polymer chemistry have led to the synthesis of various building blocks with complex architectures and functionalized properties. Such responsive building blocks that can self-organize into higher-order structures may form soft patchy particles, which have directional interactions and varying softness. Furthermore, topological effects that arise in systems with complex architecture can alone lead to a range of interesting phenomena in and out of thermodynamic equilibrium for both low [915] and high system densities [16-21]. Functionalized biomolecules such as DNA-grafted colloidal particles represent a typical example where patchiness reflects the competition between inter- and intra-particle associations [22-25]. However, despite its significance, DNA-based research is very specialized and yields limited amounts of samples. An alternative design of patchy particles is based on the so-called Telechelic Star Polymers (TSPs), that is star polymers with functionalized end groups [26-29]. A TSP consists of $f$ amphiphilic AB-block copolymer arms grafted on a common center. The solvophilic A-block is attached at the center of the star, whereas the solvophobic B-block is exposed to the exterior of the star. On changing the solvent quality through temperature variation, the outer blocks become attractive and form patches on the surface 
of the particle [30-32]. With such a TSP system, it is thus possible to cover the entire range of inter-particle interactions, from purely repulsive to attractive soft colloids simply by changing the solvent quality. The self-assembly of TSPs at the single molecule level and in concentrated solutions depends mainly on three parameters: $(i)$ the functionality $f$ of the stars, (ii) the outer block size ratio $\alpha=N_{\mathrm{B}} /\left(N_{\mathrm{A}}+N_{\mathrm{B}}\right)\left(N_{\mathrm{A}}\right.$ and $N_{\mathrm{B}}$ denote the length of the respective block), and (iii) the attraction strength between the outer solvophobic blocks which is enhanced upon worsening solvent quality.

TSPs with low functionality $(f \leq 5)$ collapse into a watermelon structure with one single patch on the surface of the particle [30]. However, more complex structures with a richer distribution of patches are formed in TSPs with higher functionality [31]. Such soft patchy particles can preserve their properties such as the size, number, and arrangement of patches upon increasing the TSP concentration [33]. The inherent flexibility of such soft-particles leads to formation of ordered structures in the case of high functionality [33]. On the other hand, low-functionally TSPs tend to form micellar aggregates [34-37], which at relatively high concentrations self-assemble into long worm-like micelles $[35,38]$. In previous work, we have examined the effects of temperature (or attraction strength) on the self-assembly of these low- $f$ TSPs in dilute solution [32]. In the present work, we extend these investigations by addressing the effects of the block size ratio and arm length on the selforganization of TSPs with $f=3$ in dilute solutions. The new results provide insight into the responsive behavior of TSPs, paving the way for the design of functional materials with tunable properties.

\section{Materials and methods}

\section{Experimental details}

Telechelic star polymers (TSPs) with three arms made of 1,4-polybutadiene (PB) as the inner A-block and polystyrene (PS) as the outer B-block were synthesized by anionic polymerization and chlorosilane chemistry using high-vacuum techniques. Detailed information on the synthesis procedure can be found in Ref. [32]. Three different TSP samples have been used in the present study. Two of them have a similar total molar mass of about $40000 \mathrm{~g} / \mathrm{mol}$, albeit a different PS weight fraction of $f_{\mathrm{PS}}=0.14$ and 0.33 . The third sample, which was used in our previous work [32], has a lower molar mass of $M_{\mathrm{W}}=26700 \mathrm{~g} / \mathrm{mol}$ and $f_{\mathrm{PS}}=0.23$. The molar mass distribution in all three TSP samples is rather narrow with the corresponding polydispersity being around $Đ=1.03$. The detailed molecular characteristics of the samples are listed in Table 1.

We have used 1-phenyldodecane as the solvent. It has a cloud-point at $53{ }^{\circ} \mathrm{C}$ for PS [39] and $22{ }^{\circ} \mathrm{C}$ for PB (the corresponding $\theta$-temperatures are expected to be slightly higher). Solutions were prepared by mixing an appropriate amount of the TSP with the solvent to reach the desired concentration. The sample degradation was inhibited by adding $0.1 \mathrm{wt} \%$ of the TSP of the antioxidant BHT (2,6-Ditert-butyl-4-methylphenol). In order to fully dissolve TSPs, methylene chloride was used as the cosolvent. Then, the cosolvent was evaporated under ambient conditions until a constant weight was achieved.

Dynamic Light Scattering (DLS) was used to investigate the dynamics and self-assembly of TSPs in dilute solutions. In DLS, the normalized autocorrelation function $G(q, t)=$ $\langle I(q, 0) I(q, t)\rangle /\langle I(q, 0)\rangle^{2}$ of the total scattered light intensity $I(q)$ at the wave vector $q=\left(4 \pi n_{0} / \lambda\right) \sin (\theta / 2)$ $\left(n_{0}\right.$ is the refractive index, $\theta$ denotes the scattering angle, and $\lambda$ is the wavelength of the incident laser beam) is related to the normalized time correlation function of the scattered electric field $E(q, t)$ by the Siegert relation:

$$
G(q, t)=1+f^{*}|\tilde{\alpha} g(q, t)|^{2}=1+f^{*}|C(q, t)|^{2},
$$

where $f^{*}$ stands for the coherence instrumental factor, $\tilde{\alpha}$ is the fraction of $I(q)$ associated with fluctuations relaxing with times longer than $0.1 \mu$ s [40-42], and $C(q, t)$ denotes the intermediate scattering function (ISF). The inverse Laplace transformation using the constrained regularization method was applied to compute the relaxation spectrum

Table 1 Molecular characteristics of investigated star diblock copolymers (PS-b-PB) 3

\begin{tabular}{lllllll}
\hline Sample & $M_{\mathrm{w}}^{\text {star }}(\mathrm{g} / \mathrm{mol})$ & $M_{\mathrm{w}}^{\text {linear }}(\mathrm{g} / \mathrm{mol})$ & $f_{\mathrm{PS}} \%(\mathrm{w} / \mathrm{w})$ & $f_{\mathrm{PB}} \%(\mathrm{w} / \mathrm{w})$ & $c / c^{*}$ & $n\left(\mathrm{~mol} / \mathrm{m}^{3}\right)$ \\
\hline 1 & 40500 & 13000 & 0.14 & 0.86 & 0.03 & 0.14 \\
2 & 40000 & 13000 & 0.33 & 0.67 & 0.04 & 0.11 \\
3 & 26700 & 8800 & 0.23 & 0.77 & 0.05 & 0.31 \\
\hline
\end{tabular}

$M_{\mathrm{w}}^{\text {star }}$ is the total molar mass of the TSP, $M_{\mathrm{w}}^{\text {linear }}$ is the arm molar mass, $f_{\mathrm{PS}}$ and $f_{\mathrm{PB}}$ are the weight fractions of PS and PB blocks, respectively. $c$ is the TSP concentration in terms of the overlap concentration $c^{*}=3 M_{\mathrm{w}}^{\text {star }} /\left(4 \pi R_{\mathrm{h}}^{3} \mathcal{N}_{\mathrm{A}}\right)\left(R_{\mathrm{h}}\right.$ is the star's hydrodynamic radius at high temperatures and $\mathcal{N}_{\mathrm{A}}$ is the Avogadro number), and $n$ is the number density of TSPs in solution 
$H(\ln \tau)$. This method assumes that $C(q, t)$ can be expressed as the superposition of exponentials:

$C(q, t)=\int_{-\infty}^{+\infty} H(\ln \tau) \exp (-t / \tau) \mathrm{d}(\ln \tau)$

The characteristic relaxation times correspond to the peak positions of $H(\ln \tau)$, whereas the area under the peak defines the value of $\tilde{\alpha}$ in Eq. 1 and hence the intensity $\tilde{\alpha} I(q)$ associated with the particular dynamic process. The transformation was performed with the program CONTIN [43] that yielded the relaxation time and intensity of the partitioning modes.

DLS experiments were performed on an ALV-5000 goniometer/correlator setup (ALV-GmbH, Germany). The light source was a Nd:YAG dye-pumped, air-cooled laser $(100 \mathrm{~mW})$ with the wavelength $\lambda=532 \mathrm{~nm}$. The refractive index of 1-phenyldodecane is $n_{0}=1.482$. Before each DLS experiment, the samples were equilibrated at $T=$ $60{ }^{\circ} \mathrm{C}$, which is above the cloud point of the outer PSblock, for $10 \mathrm{~min}$ to erase thermal history. Then, the sample was quenched to the desired temperature and equilibrated. The equilibration process was probed by measuring the ISF until it reached steady values over time. The duration of equilibration depended on the temperature and ranged from $10 \mathrm{~min}$ for $T=60^{\circ} \mathrm{C}$ to $10 \mathrm{~h}$ for $T=20^{\circ} \mathrm{C}$.

\section{Simulation details}

To model TSP dynamics under worsening solvent conditions for its outer block, we have employed a coarsegrained dissipative particle dynamics (DPD) model with explicit solvent. In what follows, the inner blocks of a TSP are labeled "A," the outer ones_- "B," whereas solvent particles_- "S." In DPD, the total force $\mathbf{F}_{i}$ acting on the $i$ th particle is composed of the conservative $\mathbf{F}^{\mathrm{C}}$, dissipative $\mathbf{F}^{\mathrm{D}}$, and random $\mathbf{F}^{\mathrm{R}}$ contributions [44]:

$\mathbf{F}_{i}=\sum_{j \neq i}\left(\mathbf{F}_{i j}^{\mathrm{C}}+\mathbf{F}_{i j}^{\mathrm{D}}+\mathbf{F}_{i j}^{\mathrm{R}}\right)$.

In Eq. 3 above, $\mathbf{F}_{i j}^{\mathrm{C}}$ is the conservative force acting between the $i$ th and $j$ th particle separated by a distance $r_{i j}$ (here and in what follows, $\mathbf{r}_{i j}=\mathbf{r}_{i}-\mathbf{r}_{j}, r_{i j}=\left|\mathbf{r}_{i j}\right|, \hat{\mathbf{r}}_{i j}=\mathbf{r}_{i j} / r_{i j}$, and $\left.\mathbf{v}_{i j}=\mathbf{v}_{i}-\mathbf{v}_{j}\right)$ :

$\mathbf{F}_{i j}^{\mathrm{C}}=A_{i j} w\left(r_{i j}\right)$,

where $A_{i j}$ is the maximal repulsion between the particles and $w\left(r_{i j}\right)$ is given by

$w\left(r_{i j}\right)=\left(1-r_{i j} / r_{\text {cut }}\right) \theta\left(r_{\text {cut }}-r\right)$,

with $\theta(x)$ denoting the Heaviside step function and the cutoff distance $r_{\text {cut }}$ being chosen as the unit of length $\left(r_{\text {cut }}=\right.$ 1). Furthermore, $\mathbf{F}_{i j}^{\mathrm{D}}$ is the pairwise dissipative force

$\mathbf{F}_{i j}^{\mathrm{D}}=-\gamma w\left(r_{i j}\right)^{2}\left(\hat{\mathbf{r}}_{i j} \cdot \mathbf{v}_{i j}\right)$, and $\mathbf{F}_{i j}^{\mathrm{R}}$ is the pairwise random force

$\mathbf{F}_{i j}^{\mathrm{R}}=-\sqrt{2 \gamma k_{\mathrm{B}} T / \Delta t} \cdot \eta_{i j} w\left(r_{i j}\right)$,

where $\eta_{i j}$ is a Gaussian random number with zero mean and unit variance. The unit of mass is set by the (same) mass of every particle $m$, whereas the unit of energy was chosen to be $k_{\mathrm{B}} T\left(k_{\mathrm{B}} T=1\right)$. The simulation were performed using the HOOMD-blue simulation package [45-48] using friction coefficient $\gamma=4.5 m \tau^{-1}$ and the equations of motion were integrated using the Velocity-Verlet algorithm [49] with time step $\Delta t=0.04 \tau$, where $\tau=r_{\text {cut }} \sqrt{m / k_{\mathrm{B}} T}$ is the DPD unit of time.

To obtain the total polymerization degree $N$ of the star arms in the experimental samples considered, we first estimated the molar volumes of $\mathrm{PB}$ and $\mathrm{PS}$, given by $v_{\mathrm{PS} / \mathrm{PB}}=M_{\mathrm{W}} / \rho_{\mathrm{PS} / \mathrm{PB}}$, where $\rho_{\mathrm{PS} / \mathrm{PB}}$ is the corresponding molar density $\left(\rho^{\mathrm{PS}}=1.05 \mathrm{~g} / \mathrm{mL}\right.$ and $\left.\rho^{\mathrm{PB}}=0.892 \mathrm{~g} / \mathrm{mL}\right)$. $N$ and $\alpha_{\mathrm{PS} / \mathrm{PB}}$ were then computed on the basis of the PS reference segment volume $99.2 \mathrm{~mL} / \mathrm{mol}$ and are listed in Table 2. In general, we are interested in the behavior of experimental systems in a rather narrow temperature range $20{ }^{\circ} \mathrm{C}<T<60{ }^{\circ} \mathrm{C}$, where the Flory-Huggins incompatibility parameter $\chi_{\mathrm{PS}-\mathrm{PB}}=18.78 / T-9.6 \cdot 10^{-4}$ [50] does not change substantially (experimental values of $\chi_{\text {PS-PB }} N$ for the three samples in such temperature range are $\chi_{\text {PS-PB }} N \lesssim 10$ ), implying that the self-assembly is mainly controlled by the solvent selectivity towards the outer block.

Given the computational cost of simulations with explicit solvent particles, we focused on a star polymer model with $f=3$ arms containing $N=64$ monomers, and systematically varied the outer block ratio $\alpha$ by changing $N_{\mathrm{A}}$ and $N_{\mathrm{B}}$. All our simulations were performed at total particle density $\rho r_{\text {cut }}^{3}=3$. Bonded interactions were given by $V_{\text {bond }}(r)=\frac{K}{2}\left(r-r_{\text {cut }}\right)^{2}$ with $K=50 k_{\mathrm{B}} T$. The central particle, to which all arms were connected to, was treated as a monomer of type A. In DPD, repulsion amplitudes $A_{i j}$ can be directly related to the Flory-Huggins $\chi_{i j}$ parameters [51]:

$A_{i j} \approx A_{i i}+\kappa(\rho) \chi_{i j}$

where $\kappa(\rho)$ depends on the DPD density such that $\kappa(3)=3.49$. In all simulations, we fixed the interblock incompatibility parameter $\chi_{\mathrm{AB}}=0.23$, which corresponds to $A_{\mathrm{AB}}=25.8$ at $\rho r_{\text {cut }}^{3}=3$. Such value of $\chi_{\mathrm{AB}}$ was obtained from a conservative experimental value $\left(\chi_{\mathrm{AB}} N\right)_{\exp }=10$ by taking into account finite polymer chain length corrections: $\chi_{\mathrm{AB}}=\left(\chi_{\mathrm{AB}} N\right)_{\exp }$. $\left(1+3.9 N^{2 / 3-2 v}\right) / N$ with $N=64$ and $v=0.588$ [51]. The incompatibility parameter $\chi_{\text {AS }}$ for the outer block and effective solvent particles was always set to $\chi_{\mathrm{AS}}=0$, which corresponds to $A_{\mathrm{AS}}=25$. Moreover, $\chi_{\mathrm{BS}}$ was systematically varied between 0 and 7 with step $\approx 0.72$, corresponding to $A_{\mathrm{BS}}$ in the range between 25 
Table 2 Composition of investigated star diblock copolymers (PS-b-PB) 3

\begin{tabular}{llllll}
\hline Sample & $v_{\text {PS }}[\mathrm{L} / \mathrm{mol}]$ & $v_{\text {PB }}[\mathrm{L} / \mathrm{mol}]$ & $v_{\text {total }}[\mathrm{L} / \mathrm{mol}]$ & $\alpha_{\mathrm{PS}}$ & $\alpha_{\mathrm{PB}}$ \\
\hline 1 & 1.73 & 12.53 & 14.26 & 0.12 & 0.88 \\
2 & 4.09 & 9.76 & 13.85 & 0.30 & 0.70 \\
3 & 1.93 & 7.60 & 9.53 & 0.20 & 0.80 \\
\hline
\end{tabular}

$v_{\mathrm{PS}}$ and $v_{\mathrm{PB}}$ are molar volumes of PS and $\mathrm{PB}$, respectively, whereas $v_{\text {total }}$ is the total molar volume of the TSP. $N$ is the TSP's polymerization degree computed on the basis of PS reference segment volume, and $\alpha_{\mathrm{PS}}$ and $\alpha_{\mathrm{PB}}$ denote the resulting number fractions of the two components

and 50. Finally, note that the main goal of our simulations using such a coarse-grained model is not to quantitatively reproduce the change of star properties with increasing $\chi_{\mathrm{BS}}$, for which atomistic simulations with realistic solvent interactions would be necessary, but to qualitatively assess the effect of self-associations on the change of TSPs static and dynamic behavior.

\section{Experimental results}

We first focus on the effect of the outer PS-block fraction on the dynamic relaxation of the TSP system at low densities. In Fig. 1, we show the experimental ISFs at a fixed wave vector and various temperatures for the two samples with comparable total molar masses but with distinct PS weight fractions. The ISFs show two distinct trends upon changing temperature. At high temperatures, the ISFs show a single exponential decay that demonstrates the existence of individual stars in solution. However, when temperature is reduced below the cloud temperature of the outer-block, the ISF features a two-step decay which indicates the coexistence of two distinct populations in the system. The first decay (fast process) in the ISF is similar to the one observed at high temperatures and hence represents the

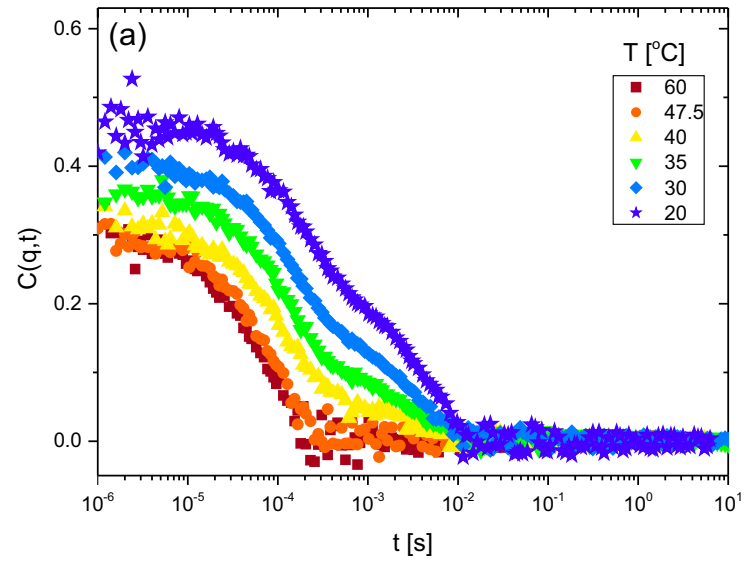

Fig. 1 Experimental ISFs at constant wave vector $q=0.02475 \mathrm{~nm}^{-1}$ and different temperatures for TSPs with the outer PS-block fractions of $f_{\mathrm{PS}}=0.14(\mathbf{a})$ and $f_{\mathrm{PS}}=0.33(\mathbf{b})$. Note that the plateau values of individual stars in solution. On the other hand, the second decay (slow process) taking place at longer times suggests the presence of larger aggregates (clusters of TSPs). The slow process becomes more pronounced as the temperature is decreased. Interestingly, the two-step decay in the ISF appears at a slightly higher temperature for the TSP with a larger PS-fraction.

To extract hydrodynamic sizes associated with the two processes in the solution, that is individual TSPs and clusters, the relaxation spectrum is calculated from the inverse Laplace transformation of the ISF using the constraint regularized method [43] discussed in Section 4. Typical results of such analysis for the TSPs with two different PS fractions at $T=60{ }^{\circ} \mathrm{C}$ and $30{ }^{\circ} \mathrm{C}$ are shown in Fig. 2. The relaxation spectrum at $60{ }^{\circ} \mathrm{C}$ shows a single peak, which is rather sharp, reflecting single exponential decay of the ISF mode. The position of the peak shifts to a slightly longer time for the TSP with a smaller PS fraction, indicating a larger hydrodynamic radius. At the lower temperature of $30^{\circ} \mathrm{C}$, the relaxation spectrum exhibits two well-separated peaks, as seen in Fig. 2b. Similarly to high temperatures, the position of the first peak shifts to a slightly longer time for the TSP with a smaller PS fraction, whereas the position of the second peak in both TSP samples is located at a

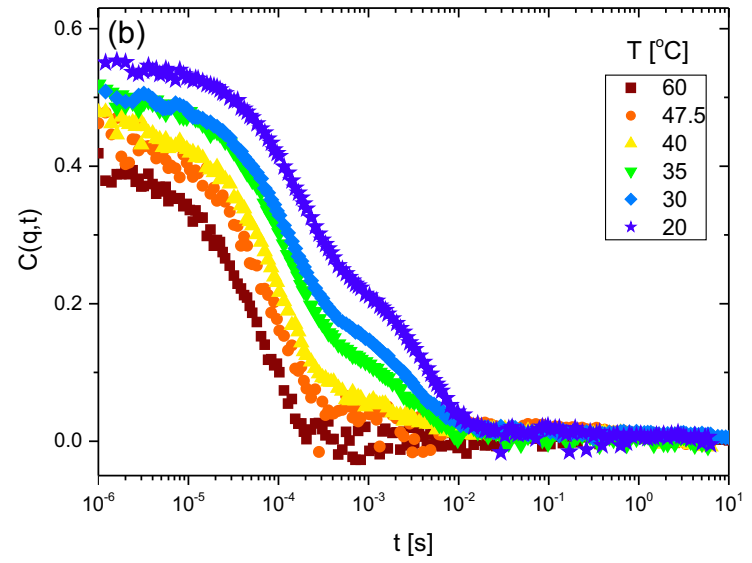

the ISF at short times are well below one. This is due to the fact that a part of the scattered intensity originates from density fluctuations of solvent molecules 


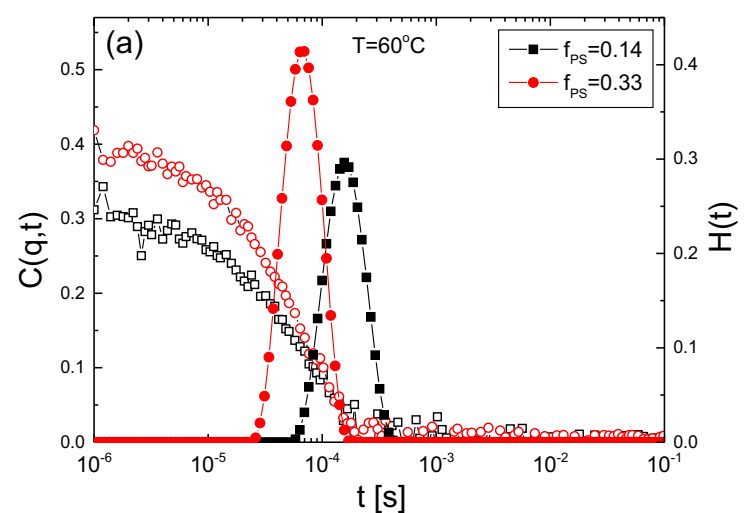

Fig. 2 ISF (open symbols, left axis) and its corresponding relaxation times spectrum (closed symbols, right axis) deduced from the constrained regularization method for the TSPs with PS fractions of $f_{\mathrm{PS}}=$

similar time. These two peaks represent the relaxation times associated with individual TSPs and clusters, respectively. Subsequently, the two relaxation times are used to calculate the diffusion coefficients associated with each component. The diffusion coefficient for the fast mode (where $q R<1$ ) is $q$-independent, whereas $D$ for the slow mode (where $q R \geq 1$ ) shows some $q$-dependence. In the latter case, $D$ extrapolated to $q=0$ is used to calculate $R_{\mathrm{h}}$. Then, the hydrodynamic sizes of TSPs and clusters are obtained using the Stokes-Einstein-Sutherland relation.

We first examine the effect of the block size ratio $\alpha$ on the single TSP size upon cooling. In experiments, the radius of gyration was too small to be probed by DLS. Instead, we focused on the hydrodynamic radius $R_{\mathrm{h}}$ of individual TSPs in dilute solution, as calculated from the fast process in the ISF using the Stokes-Einstein-Sutherland relation. The temperature dependence of $R_{\mathrm{h}}$ for the three studied TSP samples is shown in Fig. 3. The single star size exhibits a two-step shrinkage upon reducing temperature or

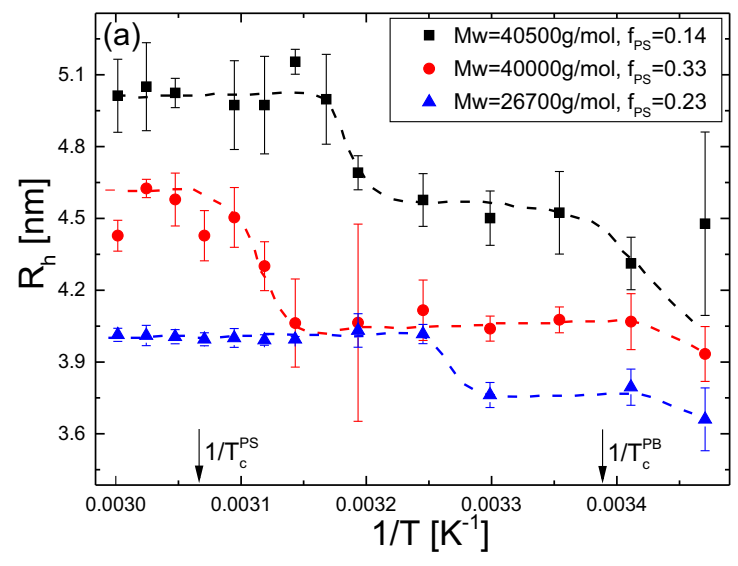

Fig. 3 a The hydrodynamic radius, $R_{\mathrm{h}}$, of individual TSPs in the dilute solution calculated from the fast process in the experimentally determined ISFs. $\mathbf{b} R_{\mathrm{h}}$ normalized by its plateau value at high temperatures.

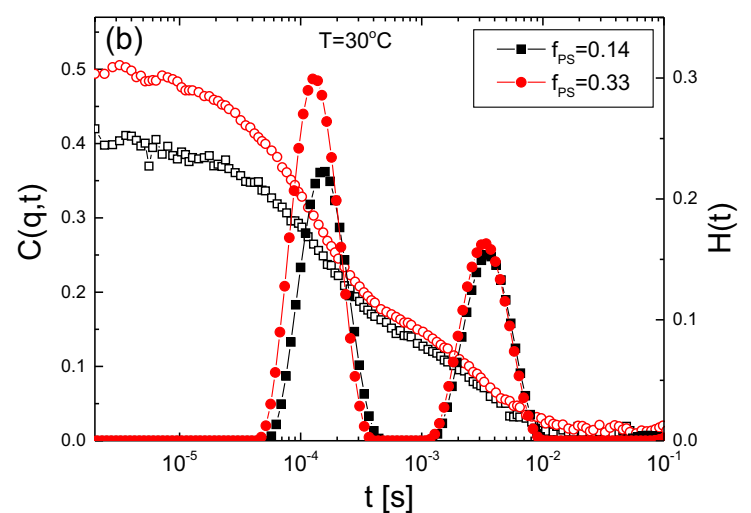

0.14 (black squares) and 0.33 (red circles) at $q=0.02475 \mathrm{~nm}^{-1}$ for $T=60{ }^{\circ} \mathrm{C}(\mathbf{a})$ and $T=30{ }^{\circ} \mathrm{C}(\mathbf{b})$

equivalently worsening the solvent quality. The first decay in size takes place at temperatures well below the cloudpoint of outer PS-blocks, whereas the second drop is seen when temperature is reduced further below the cloud-point of the inner PB-block. Hence, the first decrease in size is associated with the collapse of outer blocks, whereas the second decay corresponds to the case when inner blocks start to collapse. At high temperatures, for the TSP with $f_{\mathrm{PS}}=0.14$, we find $R_{\mathrm{h}} \approx 5 \mathrm{~nm}$ and for $f_{\mathrm{PS}}=0.33$, $R_{\mathrm{h}} \approx 4.6 \mathrm{~nm}$ (see Fig. 3a). Although both TSPs have almost the same molar mass of about $40000 \mathrm{~g} / \mathrm{mol}$, the difference in their size originates from the difference in the fraction of PS. The radius of gyration of a star homopolymer in good solvent conditions is given by $R_{\mathrm{g}} \approx \kappa(f) b N^{\nu}$, where $v=0.588, N$ is the number of Kuhn segments in a star arm, $b$ is the size of a Kuhn segment, and $\kappa(f)$, which depends on the number of star arms $f$, is a numeric constant that takes into account the star functionality [52-54]. Using the latter relation, it can be found that the size of a star made of purely

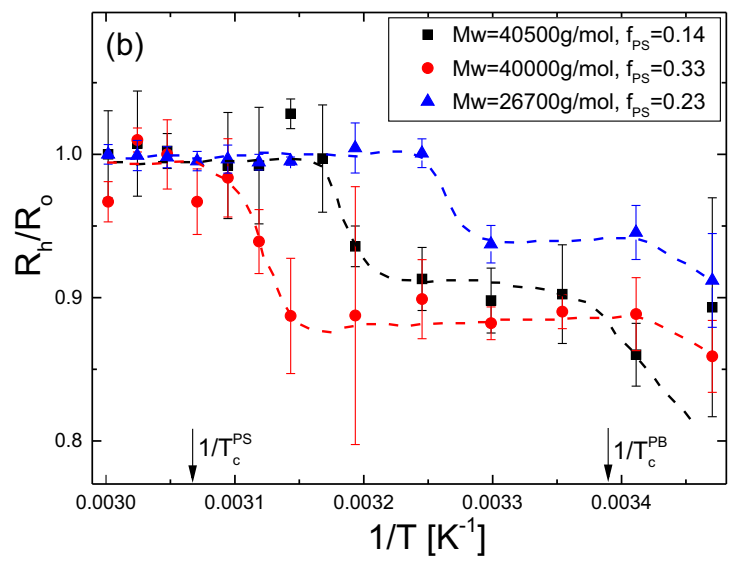

The dashed lines serve as a guide to the eye. The black arrows indicate the cloud-points of inner PB and outer PS blocks 
PB is about $65 \%$ larger compared with that made of purely $\mathrm{PS}, R_{\mathrm{g}}^{\mathrm{PB}} \approx 1.65 R_{\mathrm{g}}^{\mathrm{PS}}$, given that their molar mass is the same [55]. Hence, it is expected that the increase in PS fraction reduces the size of a TSP. In order to compare the collapse process for stars of different size, we have normalized the TSP size by the plateau value of $R_{\mathrm{h}}$ at high temperatures.

The TSP with a larger fraction of outer PS-blocks ( $f_{\mathrm{PS}}=0.33$ ) shows the first-step reduction in size at higher temperatures and the second-step drop at slightly lower temperatures compared with the TSP with a smaller PS fraction $\left(f_{\mathrm{PS}}=0.14\right)$. In both stars, the decrease in size is about 15\%, as seen in Fig. 3b. In addition, in Fig. 3, we show the results for a TSP with a smaller molar mass $(26700 \mathrm{~g} / \mathrm{mol})$ with the outer PS-block fraction $f_{\mathrm{PS}}=0.23$, which is between the other two higher molar mass TSPs with $f_{\mathrm{PS}}=0.14$ and 0.33 . The main difference is that the TSP with the smaller molar mass exhibits the decay in size at a much lower temperature compared with the other two, which originates from a smaller value of the incompatibility parameter $\sim \chi_{\text {PS-S }} N$. Moreover, the decrease in size is also rather weaker (about 10\%), which can be attributed to a shorter length of its arms.

In Fig. 4, we present the temperature dependence of clusters' $R_{\mathrm{h}}$ for the systems of TSPs with the same molar mass but two different PS fractions $\left(M_{\mathrm{W}}=40000 \mathrm{~g} / \mathrm{mol}\right.$, $f_{\mathrm{PS}}=0.14$ and 0.33 ). In both TSPs, the cluster size shrinks on cooling. However, a slight but consistent increase in the cluster size is observed when temperature is further reduced below the cloud-point of the inner PB-block. Moreover, the hydrodynamic cluster size does not show change with the fraction of outer PS block. In Fig. 4a, we additionally show the results for the TSP with smaller molar mass $\left(M_{\mathrm{W}}=\right.$ $26700 \mathrm{~g} / \mathrm{mol}$ ) and $f_{\mathrm{PS}}=0.23$. In this case, the temperature dependence of the cluster size is the same as for the other two TSPs $\left(f_{\mathrm{PS}}=0.14,0.33\right)$. However, the smaller molar mass TSP shows a cluster size that is nearly three times larger. This could be due to a higher concentration of these TSPs. To rule out the effect of concentration, we have normalized the cluster size by the number density of TSPs in solution (Fig. 4b). The number density takes into account for the number of stickers available in the solution. With such normalization, the differences in cluster size between different TSPs are reduced to a great extent. The minor differences could originate from the complex nature of selforganization of TSPs due to differences in their molecular characteristics.

\section{Simulation results}

We now focus on static and dynamic properties of single TSPs under worsening solvent conditions for the outer B block, that is under increasing $\chi_{\mathrm{BS}}$. To do so, we simulated single stars with $f=3$ arms of length $N=64$ using a coarse-grained DPD model with explicit solvent particles, as described in detail in Section 4. The outer block ratio was systematically varied from 0.1 to 0.5 with step 0.1 . The exact number of A- and B-type monomers in an arm was $N_{\mathrm{A}}=58,52,45,39,32$ and $N_{\mathrm{B}}=6,12,19,25$, 32 , respectively (the corresponding $\alpha=0.1,0.2,0.3,0.4$, $0.5)$. For each state point $\left(\alpha, \chi_{\mathrm{BS}}\right)$, we performed $10-12$ independent simulation runs of length $10^{5} \tau$, followed after a shorter equilibration period of $10^{4} \tau$. Single TSPs were simulated in a box of size $L=30 r_{\text {cut }}$ at the total particle density $\rho r_{\text {cut }}^{3}=3$. To check if such box size is sufficient to accommodate a TSP, we initially simulated the same star in good solvent conditions $\left(\chi_{\mathrm{BS}}=0\right)$ in a larger box with $L=35 r_{\text {cut }}$, and we did not observe any substantial changes
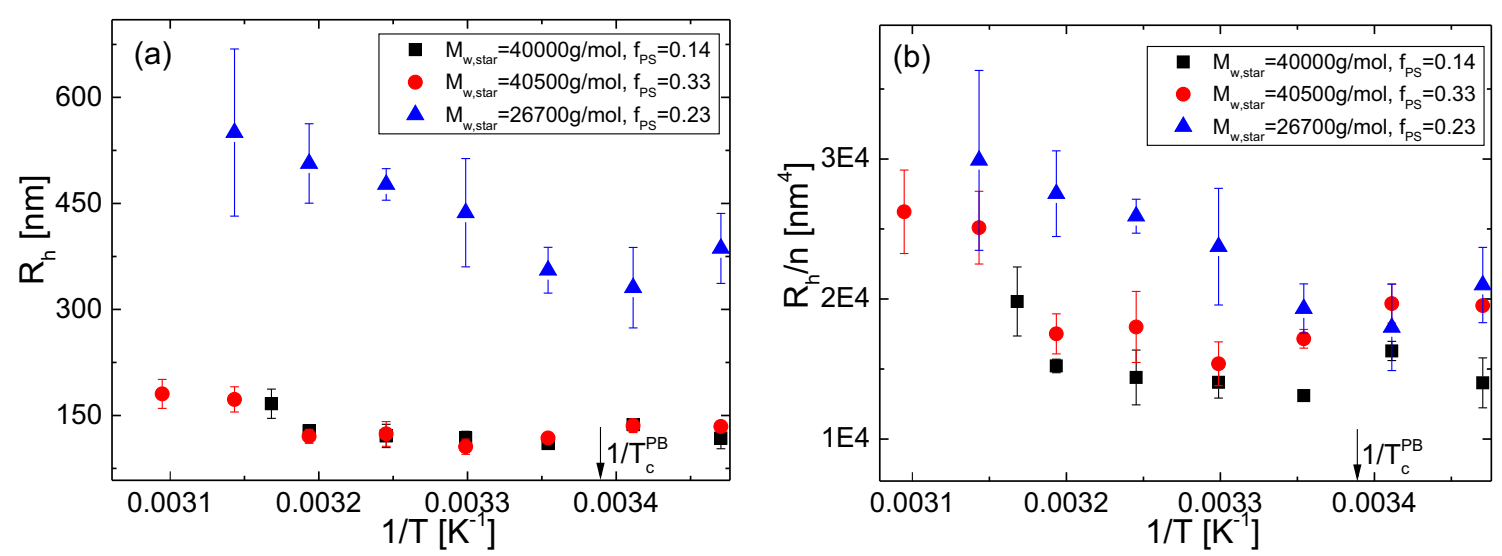

Fig. 4 a The hydrodynamic radius, $R_{\mathrm{h}}$, of clusters extracted from the slow process in the experimental ISFs. $\mathbf{b}$ The ratio of $R_{\mathrm{h}}$ to the number density $n$ (see Table 1) of TSPs in the solution. The black arrow indicates the cloud-point of inner PB blocks 

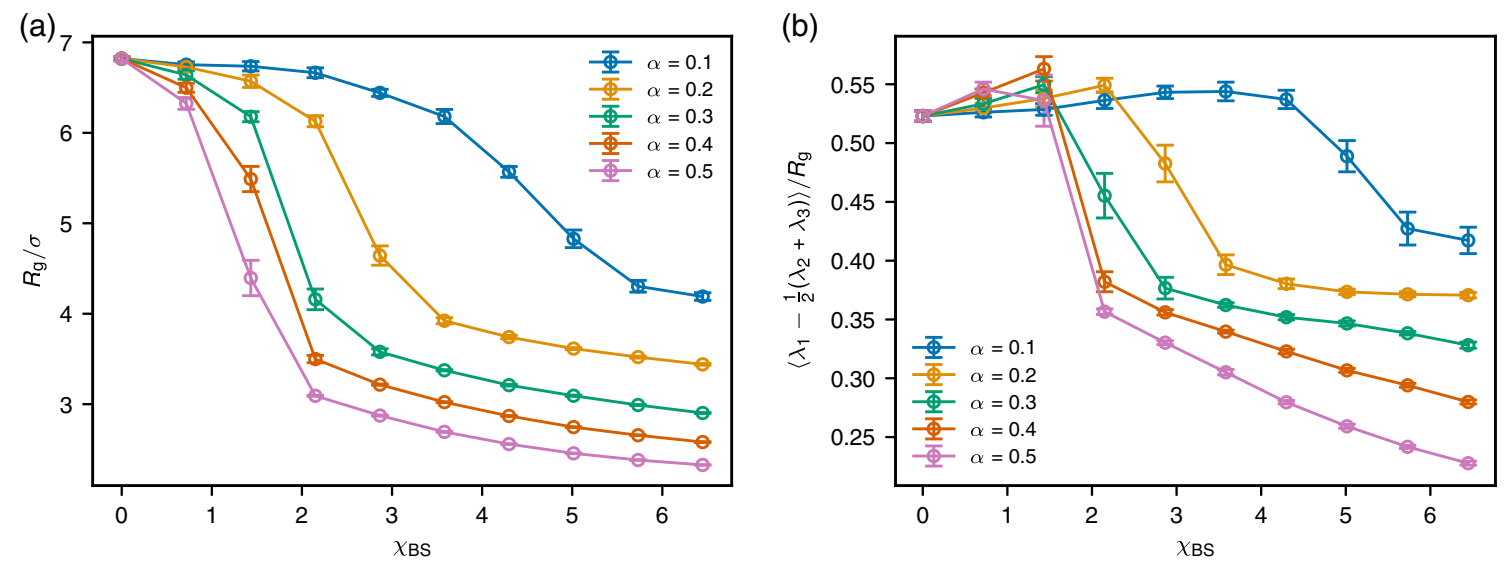

Fig. 5 a The mean radius of gyration of a TSP $R_{\mathrm{g}}$ as a function of $\chi_{\mathrm{BS}}$ for different fractions of the outer block $\alpha=N_{\mathrm{B}} / N$. $\mathbf{b}$ The mean asphericity of a TSP, computed as $\left\langle\lambda_{1}-\frac{1}{2}\left(\lambda_{2}+\lambda_{3}\right)\right\rangle$, scaled with its mean radius of gyration $R_{\mathrm{g}}$ as a function of $\chi_{\mathrm{BS}}$ for different $\alpha$

in its properties. In selective solvents, the TSP size is even smaller due to the formation of patches, which justifies the use of the same box size $L=30 r_{\text {cut }}$ in this case.

To assess single star shape properties, we computed the eigenvalues $\lambda_{i}\left(i=1,2,3, \lambda_{1} \geq \lambda_{2} \geq \lambda_{3}\right)$ of the star's gyration tensor

$G_{i j}=\frac{1}{f N+1} \sum_{k=1}^{f N+1} \Delta r_{i}^{(k)} \Delta r_{j}^{(k)}$,

where $\Delta r_{i}^{(k)}$ is the $i$ th component of the $k$ th monomer's position in the star's center of mass frame. In Fig. 5, we report the TSP's mean radius of gyration $R_{\mathrm{g}}=\left\langle R_{\mathrm{g}}^{2}\right\rangle^{1 / 2}$ $\left(R_{\mathrm{g}}^{2}=\lambda_{1}+\lambda_{2}+\lambda_{3}\right)$ as well as the mean asphericity parameter $\left\langle\lambda_{1}-\frac{1}{2}\left(\lambda_{2}+\lambda_{3}\right)\right\rangle$, which is positive and can vanish only for a completely symmetric configuration, as a function of $\chi_{\mathrm{BS}}$ for different block length ratios $\alpha$. The angles $\langle\cdots\rangle$ denote an ensemble and time average. We find that the behavior of a single TSP size is generally very similar to the experimental one (see Fig. 3): upon increasing $\chi_{\mathrm{BS}}$, we first observe a rather small decrease in $R_{\mathrm{g}}$, followed by a major drop at higher $\chi_{\mathrm{BS}}$. Such behavior of $R_{\mathrm{g}}$ is associated with the formation of a single patch, where all three arms of a TSP clump together (see Fig. 6). We find that the transition point shifts towards a higher $\chi_{\mathrm{BS}}$, that is a lower temperature because $\chi \sim 1 / T$, with decreasing $\alpha$, which is in full accordance with the experimental behavior of the two samples with $f_{\mathrm{PS}}=$ 0.14 and 0.33 that have a very similar total molar mass (see Fig. 3b). Afterwards, only a small reduction of $R_{\mathrm{g}}$ is observed upon increasing $\chi_{\mathrm{BS}}$, as seen in Fig. 3a. We also note that simulations do not capture the second drop in size which is observed in experiments for temperatures below the cloud-point of inner-block. The reason for this discrepancy is that in simulation, for simplicity reasons, the inner-block is assumed to be always in a good solvency condition. Hence, it only captures single step shrinkage process due to collapse of outer-block monomers.

We furthermore find that the final TSP size decreases with increasing $\alpha$, also in accordance with the experimental findings for the samples with $f_{\mathrm{PS}}=0.14$ and 0.33 (see Fig. $3 b$ ). This behavior is associated with generally more open configuration of collapsed TSPs with small $\alpha$ that permit solvent flow through the TSP's interior. On the other hand, in the case of larger $\alpha$, the solvophobic B-blocks form a single large patch that expels the solvent from its interior, resulting in more compact and symmetric configurations (see Figs. 6 and 5b). Interestingly, as can be seen in Fig. 5b, upon slightly increasing $\chi_{\mathrm{BS}}$ from 0 , the stars first become more aspherical, which can be attributed to the formation of transient patches between two out of three star arms. This is confirmed in Fig. 7 that reports the average number of patches formed by the star, the average number of arms in a patch, and the fraction of free arms as a function of $\chi_{\mathrm{BS}}$ for different arm compositions. In agreement with earlier results [30], we find that such TSP with $f=3$ forms only one patch for all $\alpha$ with all three arms contributing to it at high enough $\chi_{\mathrm{BS}}$. In addition, as seen from Fig. $7 \mathrm{a}$ and $7 \mathrm{~b}$, the point when all arms start to form a single patch
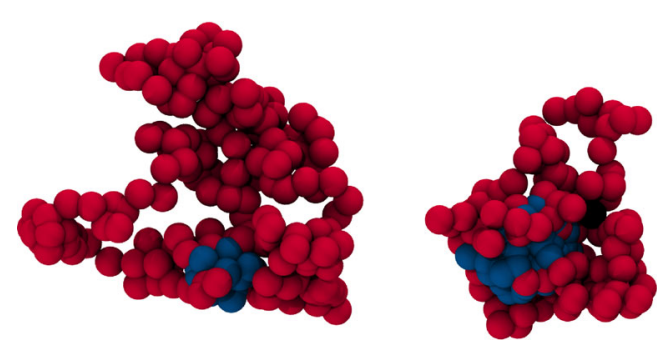

Fig. 6 Characteristic TSP conformations with $\alpha=0.1$ (left) and $\alpha=$ 0.3 (right) at a high $\chi_{\mathrm{BS}} \approx 7$. B-monomers are blue, A-monomersred, and star centers are black. Solvent particles are not shown for clarity 

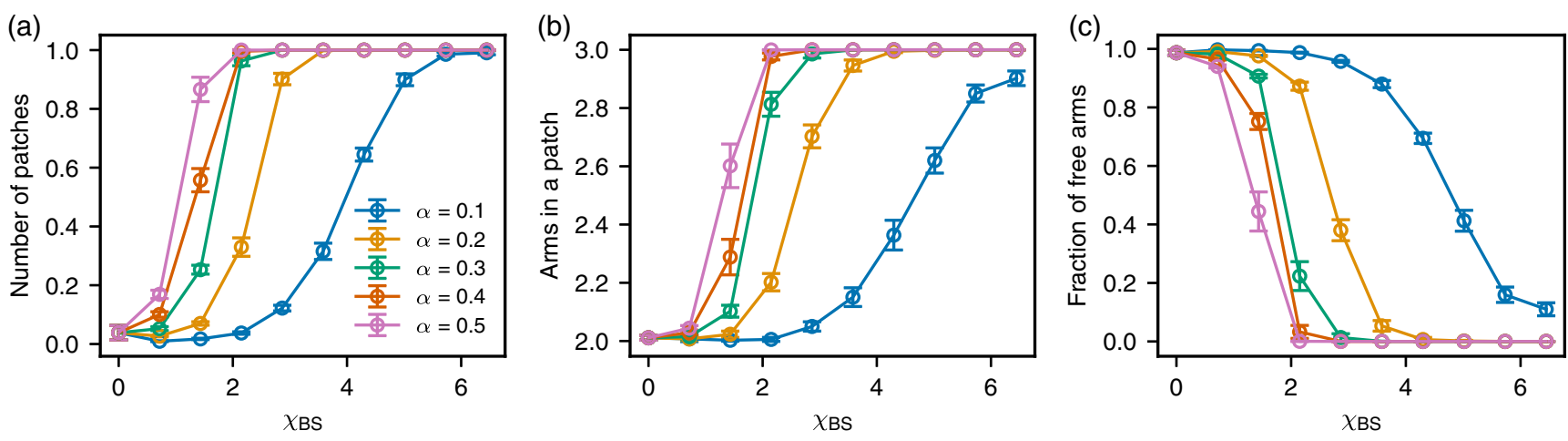

Fig. 7 a The total number of formed patches as a function of $\chi_{\mathrm{BS}}$ for different $\alpha$. Two arms are defined as being in a common patch if there is at least one pair of monomers from the two distinct arms lying at a

corresponds to the point when $R_{\mathrm{g}}$ drops significantly (see Fig. 5a). Finally, to assess the influence of arm length $N$ on the transition point for the watermelon-like structure formation, for $\alpha=0.3$, we additionally simulated stars with arm length $N=32,48,80$ for different values of $\chi_{\mathrm{BS}}$. The comparison between the behavior of the radius of gyration of TSPs with different $N$ for $\alpha=0.3$ is shown in Fig. 8. We find that the TSP with shorter arms features the star collapse at higher values of $\chi_{\mathrm{BS}}$, which therefore corresponds to lower temperatures in the experiments, being in line with the trend observed for the experimental sample with $f_{\mathrm{PS}}=0.23$ that has a lower molar mass, see Fig. 3.

The presence of faithful hydrodynamic interactions in DPD allows us to assess the influence of patch formation on the dynamics of single stars in solution. We did this by considering the mean-square displacement of TSP's center of mass, computed as:

$\operatorname{MSD}(t)=\frac{1}{T-t} \int_{0}^{T-t}\left\langle\left[\mathbf{R}\left(t^{\prime}+t\right)-\mathbf{R}\left(t^{\prime}\right)\right]^{2}\right\rangle \mathrm{d} t^{\prime}$,

where $\mathbf{R}(t)$ is the position of the star's center of mass at time $t, T$ is the total simulation time, and $\langle\cdots\rangle$ stands for the average over independent simulations runs. Typical behavior of the MSD for different $\alpha$ as well as the extracted diffusion coefficients $D$ is shown in Fig. 9. We find that the tendency to form patches, causing more compact watermelon-like structures, increases the diffusivity of the TSP. In the experiments, this behavior corresponds to a reduction in the hydrodynamic radius $R_{\mathrm{h}}$, which is in good agreement with results shown in Fig. 3. Furthermore, this effect is especially significant for the case of high $\alpha$, where $D$ at high $\chi_{\mathrm{BS}}$ can become about two times bigger compared with athermal conditions with $\chi_{\mathrm{BS}}=0$, as seen in Fig. 9a, again in agreement with the experiments, where a larger reduction in $R_{\mathrm{h}}$ is seen for the TSP with a higher PS fraction. Finally, more open conformations of collapsed TSPs with low $\alpha$ make the increase in diffusivity less pronounced (for example, about $30 \%$ increase for $\alpha=0.10$ ). distance $r \leq r_{\text {cut }}$. The average number of arms in a patch (b) and the average fraction of free arms (c) as a function of $\chi_{\mathrm{BS}}$ for different $\alpha$. $\mathbf{a}, \mathbf{b}$, and $\mathbf{c}$ share the same legend shown in (a)

In addition, we have considered the dynamics of internal patch reorganizations at the single-star level. In Fig. 10, we show the times $t_{\text {free }}$ for an arm spent in the free state, that is not forming an association with other arms, as a function of of $t_{\text {free }}$ initially decreases exponentially fast with increasing $\chi_{\mathrm{BS}}$ (Fig. 10a), up to a point where a single patch forms. At this point, $\left\langle t_{\text {free }}\right\rangle$ drops to 0 , indicating that the single patch is stable over the course of the whole simulation. The value of $\chi_{\mathrm{BS}}$ where it happens compares with the point where a significant reduction of $R_{\mathrm{g}}$ occurs (Fig. 5a). Furthermore, in Fig. 10b, we show the distribution for $t_{\text {free }}$ for $\alpha=0.1$ at various $\chi_{\mathrm{BS}}$, featuring tails that decay exponentially fast with increasing $t_{\text {free }}$ in all cases.

Finally, we consider the formation of inter-star aggregates in the dilute solution. To asses such behavior, it is necessary to simulate a sufficiently large number of stars, which becomes computationally restrictive if using the model with

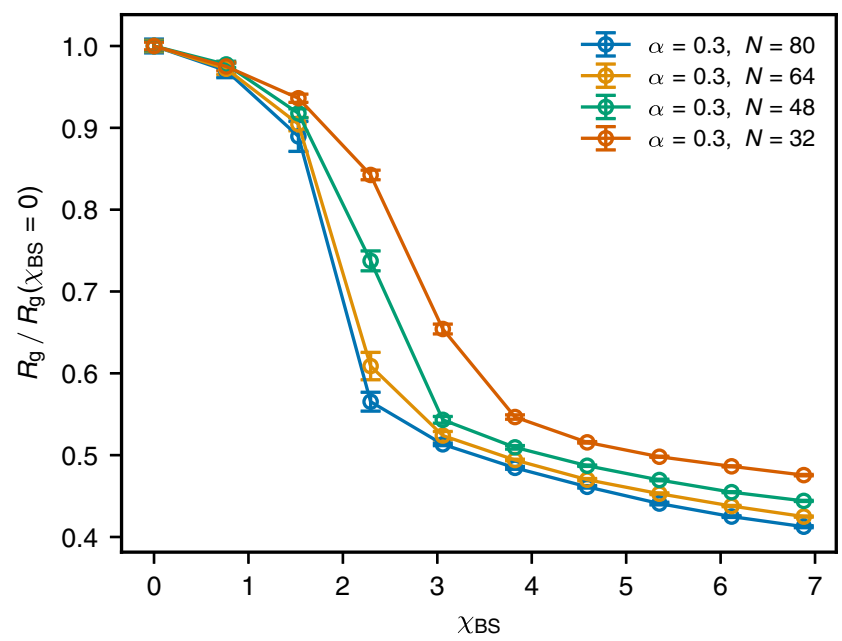

Fig. 8 The mean radius of gyration $R_{\mathrm{g}}$ of a TSP as a function of $\chi_{\mathrm{BS}}$ for the same fraction of the outer block $\alpha=0.3$ but different arm lengths $N$ $\chi_{\mathrm{BS}}$ for different $\alpha$. We find that for all $\alpha$, the mean value 
(a)

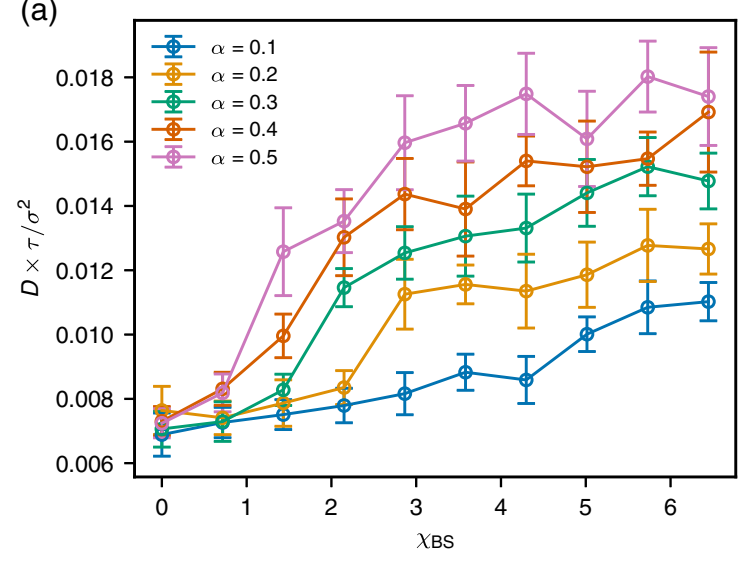

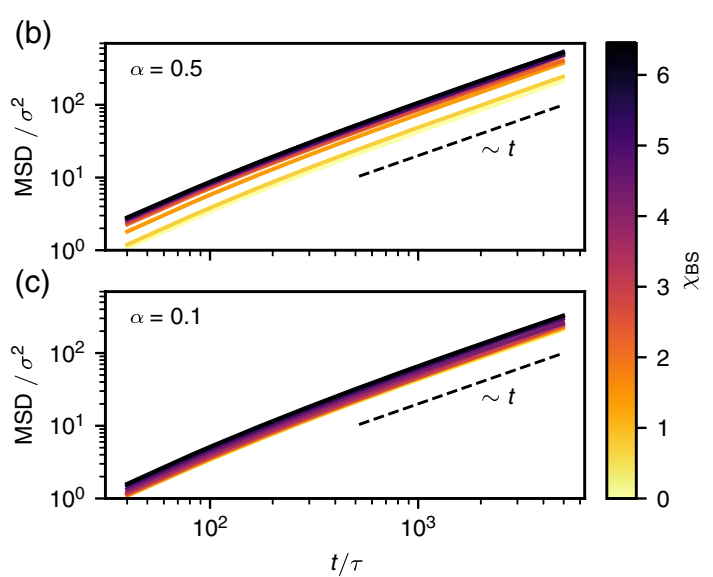

Fig. 9 a The diffusion coefficient $D$ of the TSP's center of mass as a function of $\chi_{\mathrm{BS}}$ for different $\alpha$. $D$ was extracted from the long-time behavior of the MSD of the star's center of mass, MSD $=6 D t$. Typical MSD as a function of $\chi_{\mathrm{BS}}$ are shown for $\alpha=0.5(\mathbf{b})$ and $\alpha=0.1$ (c)

$N=64$ that was employed for single star behavior discussed previously. We therefore resort to an even coarser model with, similarly, $f=3$ but with $N=10$, and in what follows we focus on the case with $\alpha=0.3$. We simulated 2000 such stars in a box with $L=70 r_{\text {cut }}$ at particle density $\rho r_{\text {cut }}^{3}=3$. In this model, the corresponding star concentration is $c \approx 0.4 c^{*}$, which is higher than the one used in the experiments (to reach an equivalent experimental concentration of $c \approx 0.04 c^{*}$, it would be necessary to simulate about a ten times bigger system containing $\sim 10^{7}$ particles). Nevertheless, even in this regime, we remain at concentrations considerably below $c^{*}$. Initially, TSPs were initialized uniformly in the box and subsequently equilibrated in athermal solvent conditions for both blocks with $\chi_{\mathrm{AS}}=\chi_{\mathrm{BS}}=0$ ( $\chi_{\mathrm{AB}}$ was set to $\chi_{\mathrm{AB}}=28.6$ to match the experimental value $\left(\chi_{\mathrm{AB}} N\right)_{\mathrm{eff}}=10$, as explained in Section 4). Afterward, the incompatibility parameter for the outer block was increased to $4.3\left(A_{\mathrm{BS}}=40\right)$ over $2 \cdot 10^{6}$ integration time steps and then further equilibrated for another $2 \cdot 10^{6}$ steps. During the latter stages, the TSPs initially began to form small micelles that subsequently merged into worm-like structures, which then again merged into a single giant cylindrical aggregate, shown in the left column of Fig. 11. Note that such cylindrical architecture is rather a consequence of periodic boundary conditions. It is likely that symmetric spherical aggregates would form in a more dilute system with a larger simulation box size, as, for instance, recently shown in Ref. [56]. Nonetheless, this illustrates the tendency of TSPs to form large aggregates even at dilute conditions, as previously shown in the experimental cluster sizes in Fig. 4. We also considered the effect of lowering temperature on the structure of such aggregate by quenching $\chi_{\mathrm{BS}}$ to $10\left(A_{\mathrm{BS}}=\right.$ 60 ) and equilibrating the system for another $10^{6}$ integration time steps. As shown in the right column of Fig. 11, as a result of the $\chi_{\mathrm{BS}}$ increase, the aggregate shrinks in the two transverse directions. This occurs because the solvophobic (a)

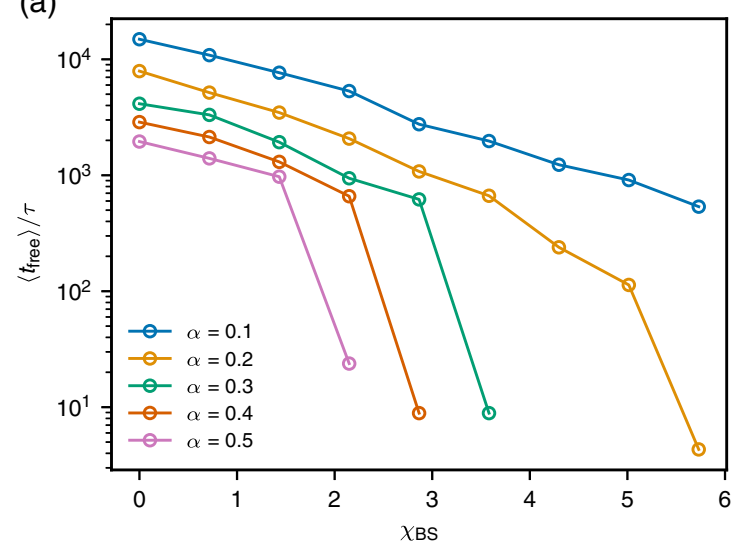

(b)

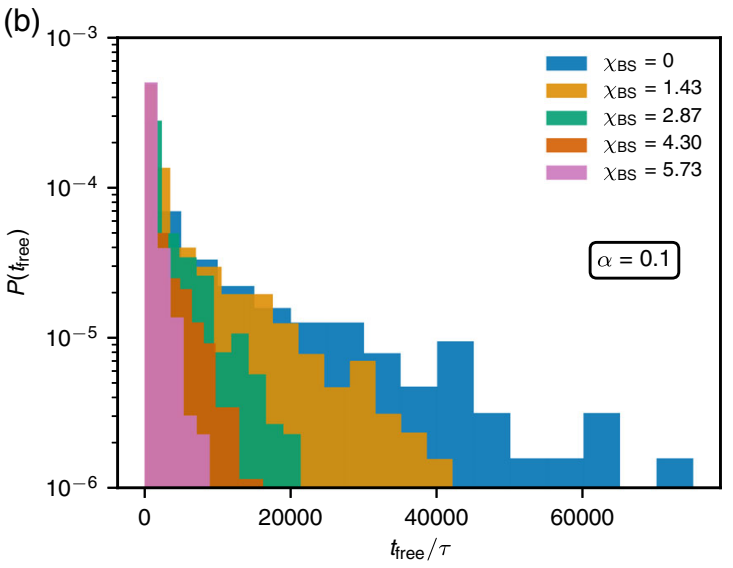

Fig. 10 a The mean time of an arm not belonging to a patch $\left\langle t_{\text {free }}\right\rangle$ as a function of $\chi_{\mathrm{BS}}$ for different $\alpha$. b The normalized distribution of $t_{\text {free }}$ for $\alpha=0.1$ and various $\chi_{\mathrm{BS}}$ 

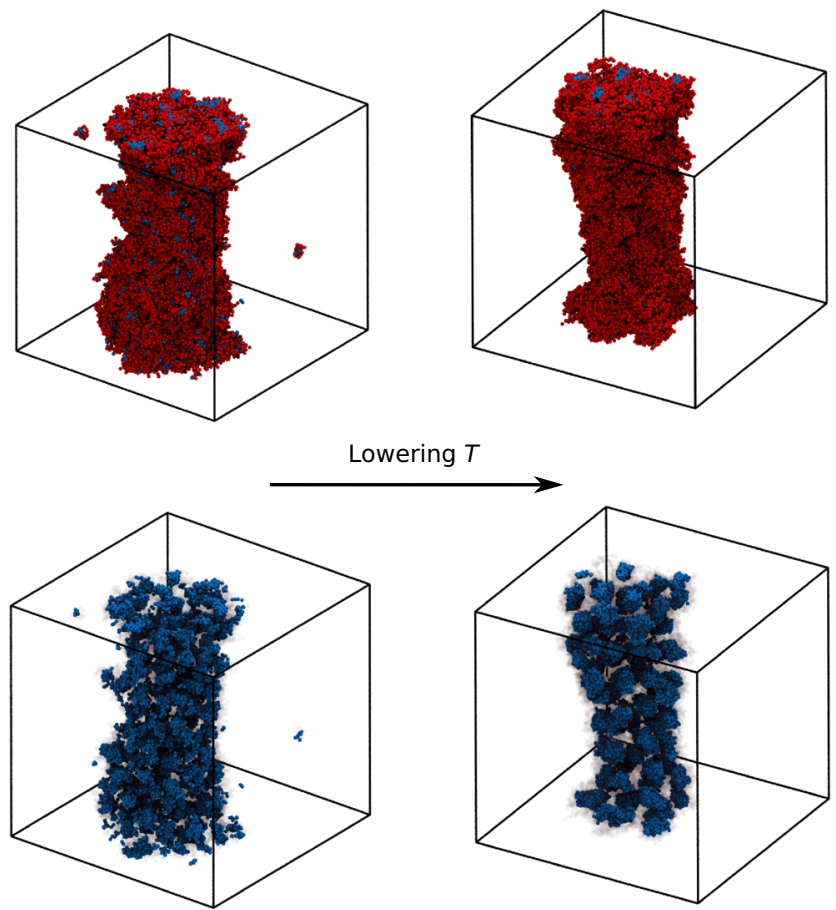

Lowering $T$

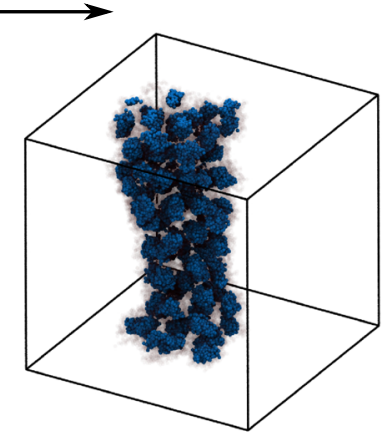

Fig. 11 Left column: self-assembly of three-arm TSPs with $N=10$ and $\alpha=0.3$ into a giant cylindrical aggregate at $c \approx 0.4 c^{*}$. The bottom row shows only the solvophobic B-blocks. Right column: the aggregate shrinks in the transverse directions upon increasing the solvophobicity of the B-monomers that become more ordered (bottom image)

TSP blocks that lie in the aggregate's interior become more ordered and thus push away the remaining solvent (see the bottom row of Fig. 11). This further agrees with the experimental trend of decreasing cluster size with decreasing temperature (Fig. 4).

\section{Conclusions}

In summary, we have investigated the self-assembly of TSPs with a variable size of the outer block as well as the arm length, which are subject to worsening solvent conditions, in dilute solutions. We find that two distinct modes in the experimental ISF appear upon lowering the temperature below a critical value: the fast-relaxing mode that corresponds to free stars in solutions as well as a slow-relaxing mode that indicates the presence of large aggregates. We find that the size of both populations decreases upon cooling. For single TSPs, the decay is associated with the formation of a single patch, where all three arms come together. From both experiments and simulations, we find that the temperature that corresponds to such transition increases with growing fraction of solvophobic monomers. However, we find that the transition temperature increases with the polymerization degree of
TSP arms, when keeping the fraction of solvophobic monomers constant. The formed aggregates in solution are much bigger than single stars $(\sim 100 \mathrm{~nm}$ versus $\sim 5 \mathrm{~nm})$. In simulations, albeit at a higher concentration of TSPs, we have found the formation of micellar aggregates with complex internal structure. Upon worsening the solvent quality for the outer block, the solvent is becoming more strongly expelled from the aggregate's interior, which causes the reduction of its size, similarly to the experimental behavior. We therefore speculate that similar objects also form at concentrations similar to the experimental ones, in line with assembled structures that have been recently observed in large-scale DPD simulations of linear diblock co-polymers in dilute conditions [56].

Acknowledgments I. C. acknowledges useful discussions with B. Capone. We are also grateful for a generous computational time at the Vienna Scientific Cluster.

Funding Open access funding provided by University of Vienna. We would like to acknowledge financial support from KAUST under grant OSR-2016-CRG5-3073-03. We also acknowledge the additional support provided by a STSM Grant from COST Action CA17139.

\section{Compliance with ethical standards}

Conflict of interest The authors declare that they have no conflict of interest.

Open Access This article is licensed under a Creative Commons Attribution 4.0 International License, which permits use, sharing, adaptation, distribution and reproduction in any medium or format, as long as you give appropriate credit to the original author(s) and the source, provide a link to the Creative Commons licence, and indicate if changes were made. The images or other third party material in this article are included in the article's Creative Commons licence, unless indicated otherwise in a credit line to the material. If material is not included in the article's Creative Commons licence and your intended use is not permitted by statutory regulation or exceeds the permitted use, you will need to obtain permission directly from the copyright holder. To view a copy of this licence, visit http:// creativecommonshorg/licenses/by/4.0/.

\section{References}

1. Pavlovic M, Antonietti M, Schmidt BV, Zeininger L (2020) J Colloid Interface Sci 575:88

2. Salonen A, Langevin D, Perrin P (2010) Soft Matter 6(21):5308

3. Vandenhaute M, Schelfhout J, Van Vlierberghe S, Mendes E, Dubruel P (2014) Eur Polym J 53:126

4. Dong J, Wang Y, Zhang J, Zhan X, Zhu S, Yang H, Wang G (2013) Soft Matter 9(2):370

5. Deng X, Livingston JL, Spear NJ, Jennings GK (2020) Langmuir 36(3):715

6. Fan TF, Park S, Shi Q, Zhang X, Liu Q, Song Y, Chin H, Ibrahim MSB, Mokrzecka N, Yang Y et al (2020) Nat Commun 11(1):1

7. Han P, Li S, Wang C, Xu H, Wang Z, Zhang X, Thomas J, Smet M (2011) Langmuir 27(23):14108

8. Balkenende DW, Monnier CA, Fiore GL, Weder C (2016) Nat Commun 7(1): 1 
9. Weiss LB, Nikoubashman A, Likos CN (2017) ACS Macro Lett 6(12): 1426

10. Chubak I, Locatelli E, Likos CN (2018) Mol Phys 116(2122):2911

11. Ahmadian Dehaghani Z, Chubak I, Likos CN, Ejtehadi MR (2020) Soft Matter 16:3029

12. Liebetreu M, Likos CN (2020) Comms Mater 1(1):4

13. Rauscher PM, Rowan SJ, de Pablo JJ (2018) ACS Macro Lett 7(8):938

14. Wu Q, Rauscher PM, Lang X, Wojtecki RJ, de Pablo JJ, Hore MJA, Rowan SJ (2017) Science 358(6369): 1434

15. Moore NT, Lua RC, Grosberg AY (2004) Proc Natl Acad Sci U.S.A 101(37):13431

16. Slimani MZ, Bacova P, Bernabei M, Narros A, Likos CN, Moreno AJ (2014) ACS Macro Lett 3(7):611

17. Weiss LB, Likos CN, Nikoubashman A (2019) Macromolecules 52(20):7858

18. Rauscher PM, Schweizer KS, Rowan SJ, de Pablo JJ (2020) Macromolecules 53(9):3390

19. Smrek J, Chubak I, Likos CN, Kremer K (2020) Nat Commun 11(1):26

20. Klotz AR, Soh BW, Doyle PS (2020) Proc Natl Acad Sci U.S.A 117(1):121

21. Rosa A, Smrek J, Turner MS, Michieletto D (2020) ACS Macro Lett 9(5):743

22. Biffi S, Cerbino R, Nava G, Bomboi F, Sciortino F, Bellini T (2015) Soft Matter 11(16):3132

23. Mirkin CA, Letsinger RL, Mucic RC, Storhoff JJ (1996) Nature 382(6592):607

24. Dreyfus R, Leunissen ME, Sha R, Tkachenko AV, Seeman NC, Pine DJ, Chaikin PM (2009) Phys Rev Lett 102(4):048301

25. Angioletti-Uberti S, Mognetti BM, Frenkel D (2012) Nat Mater 11(6):518

26. Pitsikalis M, Hadjichristidis N, Mays JW (1996) Macromolecules 29(1): 179

27. Broze G, Jérôme R, Teyssié P (1982) Macromolecules 15(3):920

28. Van Ruymbeke E, Vlassopoulos D, Mierzwa M, Pakula T, Charalabidis D, Pitsikalis M, Hadjichristidis N (2010) Macromolecules 43(9):4401

29. Vlassopoulos D, Pakula T, Fytas G, Pitsikalis M, Hadjichristidis N (1999) J Chem Phys 111(4):1760

30. Lo Verso F, Likos CN, Mayer C, Löwen H (2006) Phys Rev Lett 96:187802

31. Rovigatti L, Capone B, Likos CN (2016) Nanoscale 8:3288
32. Moghimi E, Chubak I, Statt A, Howard MP, Founta D, Polymeropoulos G, Ntetsikas K, Hadjichristidis N, Panagiotopoulos AZ, Likos CN, Vlassopoulos D (2019) ACS Macro Lett 8(7):766

33. Capone B, Coluzza I, LoVerso F, Likos CN, Blaak R (2012) Phys Rev Lett 109:238301

34. Koch C, Likos CN, Panagiotopoulos AZ, Lo Verso F (2011) Mol Phys 109(23-24):3049

35. Lo Verso F, Panagiotopoulos AZ, Likos CN (2009) Phys Rev E 79:010401

36. Lo Verso F, Panagiotopoulos AZ, Likos CN (2010) Faraday Discuss 144:143

37. Koch C, Panagiotopoulos AZ, Lo Verso F, Likos CN (2013) Soft Matter 9:7424

38. Koch C, Panagiotopoulos AZ, Lo Verso F, Likos CN (2015) Soft Matter 11:3530

39. Geerissen H, Wolf BA (1982) Chem Rapid Commun 3(1):17

40. Berne BJ, Pecora R (2000) Dynamic Light Scattering: with Applications to Chemistry, Biology and Physics (Courier Corporation)

41. Kroeger A, Belack J, Larsen A, Fytas G, Wegner G (2006) Macromolecules 39(20):7098

42. Bockstaller M, Köhler W, Wegner G, Vlassopoulos D, Fytas G (2001) Macromolecules 34(18):6359

43. Provencher SW (1982) Comput Phys Commun 27(3):229

44. Groot RD, Warren PB (1997) J Chem Phys 107(11):4423

45. Anderson JA, Lorenz CD, Travesset A (2008) J Comput Phys 227(10):5342

46. Glaser J, Nguyen TD, Anderson JA, Lui P, Spiga F, Millan JA, Morse DC, Glotzer SC (2015) Comput Phys Commun 192:97

47. Howard MP, Panagiotopoulos AZ, Nikoubashman A (2018) Comput Phys Commun 230:10

48. Phillips CL, Anderson JA, Glotzer SC (2011) J Comput Phys 230(19):7191

49. Verlet L (1967) Phys Rev 159:98

50. Wolff T, Burger C, Ruland W (1993) Macromolecules 26(7):1707

51. Groot RD, Madden TJ (1998) J Chem Phys 108(20):8713

52. Likos CN (2001) Phys Rep 348(4):267

53. Daoud M, Cotton J (1982) J Phys France 43(3):531

54. Hsu HP, Nadler W, Grassberger P (2004) Macromolecules 37(12):4658

55. Rubinstein M, Colby RH (2003) Polymer physics. Oxford University Press, New York

56. Ye X, Khomami B (2020) Soft Matter 16:6056

Publisher's note Springer Nature remains neutral with regard to jurisdictional claims in published maps and institutional affiliations. 